\title{
PECULIARITIES OF MM AND CM RADIOWAVE PROPAGATION IN THE VENUS ATMOSPHERE
}

\author{
N. N. KROUPENIO \\ Institute for Space Research, Academy of Sciences of the U.S.S.R., Moscow
}

\begin{abstract}
The total vertical absorption of $\mathrm{mm}$ and $\mathrm{cm}$ radiowaves in the Venus atmosphere is calculated from direct measurements of temperature, pressure and chemical composition, provided by the automatic interplanetary stations Venera-4, Venera- 5 and Venera- 6 . The results are in good agreement with radar measurements of Venus and with extrapolations of direct measurements of temperature and pressure. The data fit a model with surface pressure 90 atm and a water vapor content of a few milligrams per liter. Vertical radiowave attenuations at various wavelengths are computed.
\end{abstract}

The flights of the automatic interplanetary stations (AIS), Venera 4, Venera 5, and Venera 6 allowed us to carry out a series of interesting experiments in the atmosphere of Venus. AIS Venera 4, Venera 5, and Venera 6 have measured the atmospheric chemical composition, temperature, and pressure in the range of altitudes greater than $30 \mathrm{~km}$.

The space vehicle Mariner VI accomplished a radio occultation experiment at the wavelength $12.5 \mathrm{~cm}$, and from that the atmosphere altitude profile up to a pressure of about $5 \mathrm{~atm}$ has been determined. By an extrapolation of the results of Venera 4 and of Mariner $\mathrm{V}$ data into the lower altitude regions, pressure on the Venus surface was estimated, and according to the calculations turned out to be about 100 atm [1]. Values of the atmospheric parameters near the Venus surface were determined more precisely after the flights of AIS Venera 5 and Venera 6.

The results of direct measurements of the Venus atmospheric parameters allowed us to determine the conditions of propagation of $\mathrm{mm}$ and $\mathrm{cm}$ radiowaves in the planet's atmosphere. Gas analyzers installed on board Venera 5 and Venera 6 operating at $450 \mathrm{~mm} \mathrm{Hg}$ and $5 \mathrm{~atm}$ (Venera 5), and at $2 \mathrm{~atm}$ and $10 \mathrm{~atm}$ (Venera 6) have determined the following chemical composition of the atmosphere:

$$
\begin{array}{ll}
\mathrm{CO}_{2} & 97 \pm 4 \% \\
\mathrm{~N}_{2} & \text { with inert gases } \leq 2 \% \\
\mathrm{O}_{2} & \text { less than } 0.1 \% .
\end{array}
$$

Water vapor at pressures of $0.6-2.0 \mathrm{~atm}-0.6-1.1 \%$ water vapor at pressures of $5-10 \mathrm{~atm}-0.07 \%$. [2]

AIS Venera 5 and Venera 6 have measured temperatures and pressures in the altitude range greater than $30 \mathrm{~km}$ where the temperature changed from 300 to $600 \mathrm{~K}$ and pressure from 0.5 to $27 \mathrm{~atm}$ [3].

Considerable pressures and temperatures existing in the Venus atmosphere with an abundance of carbon dioxide and a small admixture of both water vapor and oxygen cause a series of interesting phenomena connected with $\mathrm{mm}$ and $\mathrm{cm}$ radiowaves propagation in the Venus atmosphere. Among these are:

- strong attenuation of radiowaves in this band, which increases with shorter wavelengths; 
- lack of a sharply prominent water vapor absorption line in the lower atmosphere;

- thermal radiation formed in the planetary atmosphere at $\mathrm{mm}$ and shortwave parts of the $\mathrm{cm}$ radioband.

For calculations of the radiowave absorption coefficient a model of the Venus atmosphere was computed based on AIS Venera 5 and Venera 6 measurement data $[2,3]$ extrapolated into the lower altitudes region under the assumption of a nearadiabatic altitude-variation of temperature.

Figure 1 gives the altitude dependences of pressure and temperature for this model. Radiowave absorption was calculated as follows. The carbon dioxide absorption coefficient wascalculated in accordance with $\mathrm{Ho}$, Kaufman and Thaddeus'semi-empirical formula, the water vapor absorption coefficient was calculated from A. P. Naumov and S. A. Jevakin's quantum-mechanical formula [5], bearing in mind the errors in our

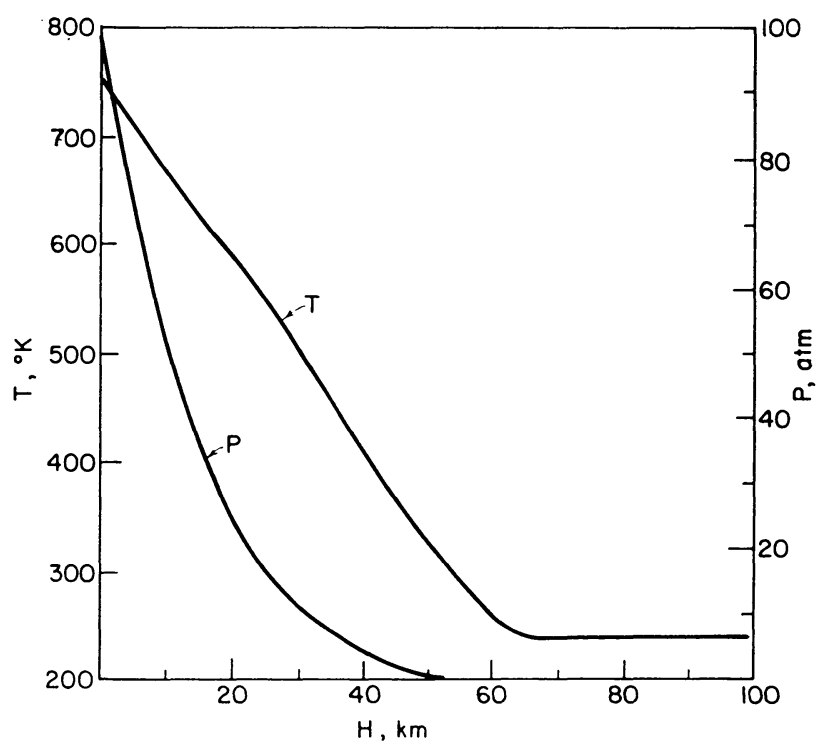

Fig. 1.

knowledge of the chemical composition of the atmosphere. As the molecular oxygen contribution to the optical thickness of Venus atmosphere beyond the $\mathrm{O}_{2}$ absorption line does not exceed $2 \%$ at the $\mathrm{mm}$ radioband and $5 \%$ at the longwave part of the $\mathrm{cm}$ radioband, an absorption due to this component was not taken into account in the calculations [6]. Absorption was calculated for an oxygen abundance of $0.4 \%$.

A complete vertical attenuation of radiowaves in the Venus atmosphere was calculated for a series of pressure values in the range of 0 to $100 \mathrm{~atm}$ and corresponding temperatures were determined by the atmosphere model considered. These calculations were performed for a carbon dioxide percentage of $97 \%$ and water vapor content of 0.1 to $0.8 \%$.

Figure 2 gives the results of the calculation for $0.1 \% \mathrm{H}_{2} \mathrm{O}$. According to these data the dependencies of complete vertical absorption of radiowaves on the trajectory 'space 
vehicle surface' were calculated as a function of the vehicle altitude above the Venus surface.

These dependencies are presented in Figure 3. Zero altitude in these calculations corresponds to a surface pressure equal to 96.7 atm [3].

From Figures 2 and 3 one can easily conclude that it is inexpedient to use wavelengths shorter than $5 \mathrm{~cm}$ both for communication between the ground control station

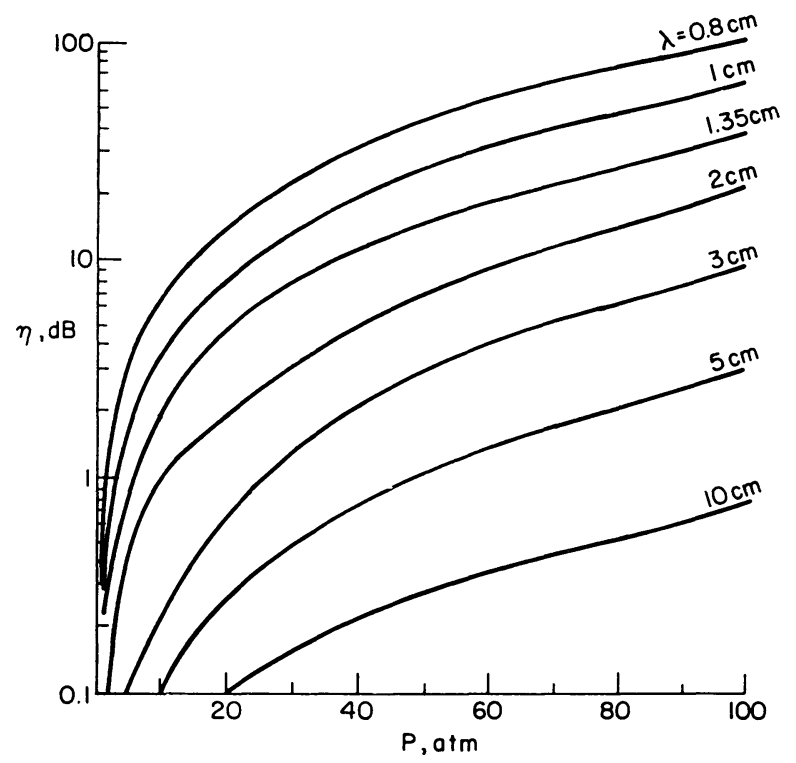

Fig. 2 .

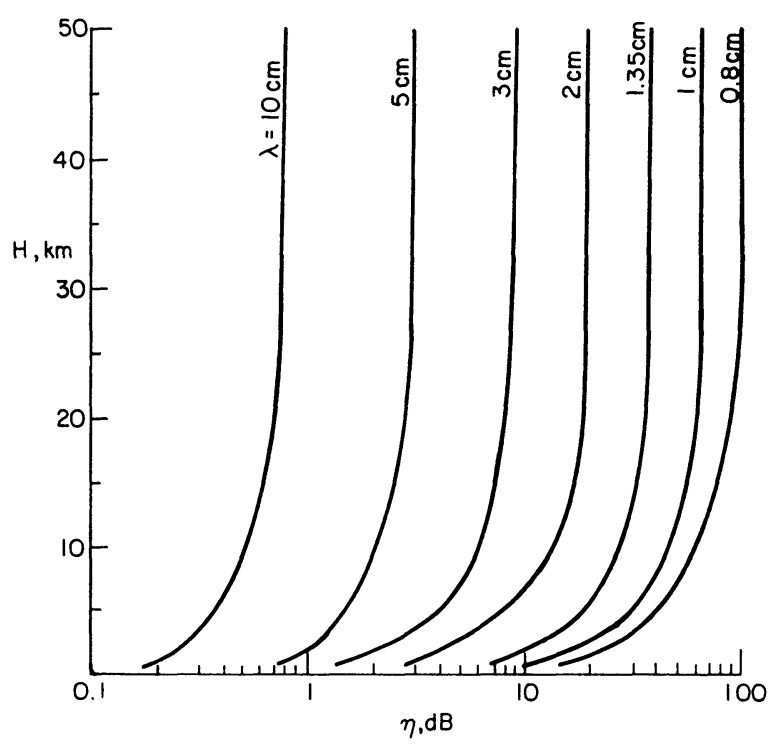

Fis. 3. 
and the space vehicle during its landing on the Venus surface, and for the operation of radiotechnical equipment of the space vehicle landing system, because with shorter wavelengths it is necessary to increase the power of the transmitters and the sensitivity of the receivers. The examination of the curves in Figure 2 shows that the values of complete vertical absorption of radiowaves with wavelengths at pressures more than $40 \mathrm{~atm}$ are described by a law close to $\lambda^{-3}$. A similar dependence is obtained in the case of sound wave propagation in an acoustically thick medium. Because of this, regularities existing in hydroacoustics can probably be applied to radiowave propagation in media with pressures more than $40 \mathrm{~atm}$. This conclusion is a preliminary one and should be verified by means of appropriate experimental studies of radiowave propagation in similar media using the experience accumulated not only by radiophysics but also by seismologists and hydroacoustic specialists.

It is of interest to compare the calculated data with the results of Venus radar measurements at wavelength $3,8,12.6$, and $23 \mathrm{~cm} \mathrm{[7,8].} \mathrm{If} \mathrm{the} \mathrm{planetary} \mathrm{radar} \mathrm{cross-}$ section is assumed to be invariant with wavelength in $\mathrm{cm}$ and $\mathrm{dm}$ bands, as can take place, for example, when the density and conductivity of the surface layer material does not vary with depth, then the measured attenuation of the reflected signal towards shorter wavelengths can be determined only by the absorption in the atmosphere.

In this case the results of Venus radar measurements from the ground stations can be correlated under the following surface pressures:

- up to 70 atm for a uniform water vapour distribution in the atmosphere at a concentration of $8 \mathrm{mg} / \mathrm{l}$.

- up to 90 atm for a uniform water vapor distribution in the atmosphere with a concentration of $1 \mathrm{mg} / \mathrm{l}$; or for water vapor with a concentration of $8 \mathrm{mg} / \mathrm{l}$ in a thin layer of the atmosphere at altitudes where the temperature does not exceed $400 \mathrm{~K}$.

- up to 110 atm when water exists only in the upper atmosphere in the solid phase.

A comparison of these three models with the results of direct measurements performed by Venera 5 and Venera 6 reveals that the best correlation occurs in the case of the second model.

Thus, a model of the Venus atmosphere obtained as a result of AIS experiments is in good agreement with the results of Venus radar measurements made from the earth.

\section{References}

[1] Kliore, A.: 1968, Report at Symposium on the Moon and Planets, Kiev, U.S.S.R.

[2] Vinogradov, A. P., Surkov, Yu. A., Andreitchikov, B. M.: 1970, Dokl. Akad. Nauk S.S.S.R. $190,552$.

[3] Avduevsky, V. S., Marov, M. Ya., Rozhdestvensky, M. K., Borodin, N. F., Koryagin, V. P.: 1970, Dokl. Akad. Nauk S.S.S.R. (in press).

[4] Ho, W., Kaufman, I. A., Thaddeus, P.: 1966, J. Geophys. Res. 71, 5091.

[5] Naumov, A. P., Jevakin, S. A.: 1968, Izv. Vysshikh. Ucheln Zavedenii, Radiofiz. 6, 674.

[6] Kroupenio, N. N., Naumov, A. P.: 1968, Report at Symposium on the Moon and Planets, Kiev, U.S.S.R.

[7] Evans, J. V.: 1965, Astron. J. 73, 125.

[8] Evans, J. V.: Report at Symposium on the Moon and Planets, Kiev, U.S.S.R. 